\title{
HERRAMIENTAS VIABILIZADORAS PARA EL ACCESO Y DIFUSIÓN DE INFORMACIÓN CIENTÍFICA
}

AUTORES: Hernández Baque Jairon Jaime ${ }^{1}$

Marcillo Merino Mario Javier ${ }^{2}$

Chancay Merchán Madileth Yolanda ${ }^{3}$

Villacreses Soledispa David Josué ${ }^{4}$

DIRECCIÓN PARA CORRESPONDENCIA: (jairo032198@gmail.com)

Fecha de recepción: 12/01/2021

Fecha de aceptación: 22/08/2021

\section{RESUMEN}

En la actualidad el internet se ha vuelto la comunidad virtual más grande de la sociedad en donde se puede alojar todo tipo de información común, pero de todo el lugar de internet solo un $2 \%$ guarda y distribuye información de carácter científico como son los Artículos Científicos, Ensayos, Ponencias, etcétera. Con el pasar de los tiempos toda la tecnología ha cambiado la forma de poder comunicarnos tanto directa como indirectamente por medio de la creación de almacenamiento rígidos para guardar información, se lo identifica como Bibliotecas virtuales, documentaciones virtuales, blocs y foros, estos medios nos auxilian a tener a mano todos los canales necesarios para poder adquirir conocimientos académicos por este motivo la tecnología en la actualidad ayuda a muchas personas a estudiar por medio de la red mas grande como es el Internet, por este motivo el articulo brinda ideas de como divulgar información de manera científica para ayudar a la sociedad a avanzar por medio de estudios, ya realizados mediante las Tecnologías de la Información "TICs” como resultados se obtendrá una colectividad del conocimiento en conjunto con el uso educado de las antes mencionadas TICs. Tomando en cuenta que el acceso al internet es cada vez más accesible provoca que las comunidades educativas tengan mayores oportunidades de obtener acceso a información valiosa pero pocos medios alojan información de carácter científico este articulo su objetivo es dar a conocer estos medios para que la comunidad de los estudiantes tenga conocimiento de dónde acudir para su debida preparación formativa.

\footnotetext{
${ }^{1}$ Estudiante de la Universidad Estatal del Sur de Manabí, Facultad de Ciencias Técnicas, Pajan, Manabí, Ecuador. hernandez-jairon4582@unesum.edu.ec. ORCID ID: https://orcid.org/0000-0003-4005-5834

${ }^{2}$ Ing. En Sistemas, MG. En docencia Universitaria, Universidad Estatal del Sur de Manabí, Jipijapa, Costa, Ecuador, E-mail: mario_marcillo_merino@hotmail.com

${ }^{3}$ Estudiante de la Universidad Estatal del Sur de Manabí, Facultad de Ciencias Técnicas, Cascol, Manabí, Ecuador. madilethchancay11@gmail.com, ORCID ID: https://orcid.org/0000-0002-0128-5752

${ }^{4}$ Estudiante de la Universidad Estatal del Sur de Manabí, Facultad de Ciencias Técnicas, Jipijapa, Manabí, Ecuador villacreses-david9703@unesum.edu.ec. ORCID ID: https://orcid.org/0000-0002-0486-2092
} 
Hernández Baque Jairon Jaime, Marcillo Merino Mario Javier, Chancay Merchán Madileth Yolanda

PALABRAS CLAVE: Artículos Científicos; Sociedad del Conocimiento; Acceso a la Información.

\section{IMPACT ON THE SOCIETY OF KNOWLEDGE IN COMPANIES}

\section{ABSTRACT}

At present, the internet has become the largest virtual community in society where all kinds of common information can be housed, but of the entire internet site only $2 \%$ save and distribute scientific information such as Scientific Articles, Essays, Presentations, etcetera. With the passing of time, all technology has changed the way we can communicate both directly and indirectly through the creation of rigid storage to store information, it is identified as virtual libraries, virtual documentation, notepads and forums, these means help us to have at hand all the necessary channels to be able to acquire academic knowledge, for this reason technology currently helps many people to study through the largest network such as the Internet, for this reason the article provides ideas on how to disseminate information in a scientific way to help society to advance through studies, already carried out through Information Technologies "ICTs", as a result, a collective of knowledge will be obtained in conjunction with the educated use of the aforementioned ICTs. Taking into account that access to the internet is increasingly accessible, it means that educational communities have greater opportunities to obtain access to valuable information but few media host scientific information, this article aims to make these media known so that the community of students have knowledge of where to go for their proper training preparation.

KEYWORDS: scientific articles; knowledge society; access to Information.

\section{INTRODUCCIÓN}

Hoy en día la tecnología está avanzando asombrosamente, sin embargo, es inevitable ponerse a la par de la misma ya que vivimos en un mundo lleno de muchas necesidades, las cuales la tecnología en gran parte las llena. Las Tics, especialmente los recursos telemáticos originan oportunidades que enriquecen el ambiente en el que se desarrolla la educación, ofreciendo nuevos servicios digitales, como los vídeos y teleconferencias, los CD-ROMs, las bibliotecas digitales, documentos y varios centros de información accesibles por medio de la Web. La sociedad emergente, es una sociedad generalizada, altamente tecnificada e interconectada y prefigura un conjunto de exigencias que deben ser satisfechas para el logro del acceso a la llamada "Sociedad del Conocimiento".

Difusión es la divulgación del conocimiento, constituye un tipo de discurso diferente, domina un conjunto de elementos o signos oportunos de un discurso especializado y una estructura que se establecen en elementos clave a la hora de su evaluación. La difusión como la divulgación científica, ambas son actividades de comunicación, puesto a que tienen en cuenta el conocimiento de las estructuras lingüísticas, el adecuado uso a las exigencias del contexto, su estructuración coherente del discurso y además el empleo de estrategias afectivas para iniciar, desarrollar y finalizar lo que es la comunicación. La competencia comunicativa considera "los conocimientos y habilidades necesarios para lograr una comunicación eficiente". (Victoria Espinosa Santos, s.f.)

90 UNESUM-Ciencias. Publicación cuatrimestral. Vol. 5, Año 2021, No. 5 (Septiembre-Diciembre) 
La presente investigación tiene por objeto dar a conocer la importancia de las herramientas viabilizadoras para el acceso y difusión de información científica además se utilizaron las metodologías de nivel teórico donde se describen los puntos más importantes de las Herramientas viabilizadoras para el acceso y difusión de Información Científica.

\section{DESARROLLO}

\section{Tecnologías de la Información}

Término que destaca en el rol de las comunicaciones unificadas y la integración de diferentes telecomunicaciones como las líneas telefónicas y señales inalámbricas, así como también un software necesario como son los middlewares, almacenamientos de sistemas audiovisuales.

Las herramientas de las Tecnologías de la Información (TIC), forman ya parte de nuestras vidas ya que las utilizamos para casi cualquier actividad. En área profesional se podría decir que se utilizan en todos los sectores, entre ellos el sector de la educación. Porque las herramientas TIC ofrecen a profesores y alumnos la posibilidad del aprendizaje colaborativo a través de Internet para brindar y difundir información científica. (Daniel Jiménez Bermejo, s.f.)

\section{El impacto de las nuevas tecnologías en la Educación.}

Las nuevas tecnologías cada día van dando un impacto mayor en la difusión de archivos Las nuevas tecnologías al pasar de los días van generando un impacto mayor en la propagación de archivos digitales para el campo académico, esto hace posible que encontremos nuevas formas de difusión de poder publicar y distribuir información científica en la World Wide web en las cuales tenemos:

\section{Libros electrónicos}

El libro electrónico no es una novedad en el ámbito académico. Su mercadeo empezó entre los años noventa junto a las revistas electrónicas. Aunque su éxito y su aceptación fueron reservados, en aquellos años se manejaba más como material complementario para la consulta y ratificación de algún dato en concreto que como materia de investigación o de estudio. Sin embargo, esta tendencia se ha modificado en parte gracias a la disponibilidad de un mayor tipo de dispositivos que facilitan y flexibilizan la experiencia de la lectura. (Stelle \& Woodward, 2009)

\section{Ordenadores y teléfonos móviles inteligentes}

Estas herramientas suelen ser de gran importancia al momento de compartir y visualizar información de una manera masiva que suele, aunque con el avance tecnológico nos permite adquirir conocimientos científicos. (navarra.es, 2018)

\section{Cursos MOOC}

Son los Massive Open On-line Course, que apoyan a que muchas más personas alcancen acceder a formación y oprimir la brecha educativa debido a sus tipologías: son gratuitos, colectivos, permiten que el alumnado pueda ubicar libremente de los contenidos y no requieren prueba de acceso.

\section{Herramientas Web para la difusión de Información Científica}

Las herramientas web permiten compartir su análisis con otros usuarios de diferentes partes del mundo gracias a diferentes servidores a nivel mundial para la creación, distribución y edición por 
Hernández Baque Jairon Jaime, Marcillo Merino Mario Javier, Chancay Merchán Madileth Yolanda

medio de fuentes Bibliográficas para ello tenemos herramientas de Tecnología de la Información para creación de blogs.

\section{Herramientas TIC para creación de blogs}

En el ámbito educativo, los blogs se han hecho rápidamente con un lugar destacado, sobre todo por su excepcional capacidad para publicar contenido e integrar recursos de muy diversas procedencias, especialmente aquellos procedentes de los servicios de la denominada Web $2.0 \mathrm{o}$ web social: audio, vídeo, animaciones, documentos, presentaciones, mapas, encuestas, líneas de tiempo, mapas conceptuales, ejercicios interactivos, etc. Para ello encontramos los siguientes servidores como son WordPress, Blogger, Tumblr, wiki en otros. (Ch., 2005)

\section{Tipos de Información Científica}

En la actualidad encontramos diferentes artículos científicos por la necesidad que se tiene por descubrir nuevas técnicas y conocimientos de diferentes ramas de la Educación por este motivo tenemos cuatro enfoques como son:

1. De acuerdo al objetivo.

2. De acuerdo a los datos empleados.

3. De acuerdo a las preparaciones sobre el objeto de estudio.

4. De acuerdo al grado de administración de las versátiles.

\section{De acuerdo al objetivo.}

1. Investigación aplicada: Se emplea para la resolución práctica de problemas específicos como enfermedades, mejoras en la producción agrícola, energía del hogar, etcétera.

2. Investigación básica: Surge a partir del interés por una determinada pregunta científica, con lo cual su objetivo principal es la obtención de un conocimiento, más allá de su practicidad en resolución de problemas. Algunos ejemplos son: ¿Cómo se generó el universo? ¿Cómo funcionan las células? entre otras.

\section{De acuerdo a los datos empleados.}

1. Investigación cualitativa: Investiga fenómenos profundos y complejos para su cuantificación matemática como es el caso de la religión, la cultura, las creencias, los modos de vida, etcétera.

2. Investigación cuantitativa: Refiere a estudios empíricos y sistemáticos que pueden ser medibles con el fin de desarrollar modelos matemáticos, teorías, hipótesis, etcétera.

\section{De acuerdo a los conocimientos sobre el objeto de estudio.}

1. Investigación explicativa: Intenta justificar por qué ocurre el hecho o fenómeno estudiado, las condiciones de su manifestación y las relaciones entre variables. Es más estructurado que otros tipos de investigación científica ya que implica pasos concretos como explorar, describir, correlacionar.

2. Investigación descriptiva: Conocida como investigación estadística busca generar un estudio preciso y concreto del objeto o fenómeno a analizar.

3. Investigación exploratoria: Se emplea para mejorar un problema que en su momento ha resultado incompleto o no ha sido definido con claridad.

De acuerdo al grado de manipulación de las variables.

92 UNESUM-Ciencias. Publicación cuatrimestral. Vol. 5, Año 2021, No. 5 (Septiembre-Diciembre) 
1. Investigación experimental: Su objetivo es ser sistemática, concreta y objetiva con el fin de predecir fenómenos mediante probabilidades y causalidades entre las variables que han sido seleccionadas para el estudio. Es el clásico enfoque de causa y efecto.

2. Investigación no experimental: Se limita a observar sin manipular los fenómenos para luego analizarlos.

3. Investigación correlacional: No determina necesariamente una relación entre la causa y efecto del fenómeno observado. Se focaliza en la correspondencia entre variables y las averigua sistémica y empíricamente. (UNI>ERSIA, 2019)

\section{HERRAMIENTA TECNOLÓGICAS PARA SU PUBLICACIÓN Y SU LECTURA EL FORO ELECTRÓNICO}

Es un instrumento tecnológico para proporcionar el aprendizaje colaborativo.

En el marco de las Tecnologías de la Información y la Comunicación están naciendo nuevas formas de proyectar y desarrolla

r los procesos de Enseñanza Aprendizaje, de modo que se comience la colaboración sincrónica y asincrónica entre los sujetos que no se localizan físicamente en el similar lugar, ni en los mismos ejes temporales.

Una de las herramientas técnicas que beneficia la interacción a distancia y asincrónica, es el Foro Electrónico o newsgroup, el cual cede la discusión entre otras personas, sobre un argumento específico.

Esta herramienta obtiene amplia utilidad, si es manejada con propiedad pedagogía, para ayudar al aprendizaje colaborativo, parte que aprueba la notificación y la interacción entre un conjunto de personas en la indagación de objetivos que le son frecuentes.

En el foro nombre que evoca las grandes asambleas romanas tienen la oportunidad de participar todos los presentes en una reunión, organizada para tratar o debatir un tema o problema determinado, con la finalidad de permitir la libre expresión de ideas y opiniones a todos los integrantes de un grupo, bajo un clima informal de mínimas limitaciones.

Dentro de su manifiesta informalidad, el foro exige un mínimo de previsiones o normas a las cuales debe ajustarse todo el grupo: tiempo limitado para cada expositor, no apartarse del tema, exponer con la mayor objetividad posible, levantar la mano para pedir la palabra, centrarse en el problema y evitar toda referencial personal.

El foro ofrece varios beneficios, entre los cuales se destacan:

- Obtener las opiniones de un grupo más o menos numeroso acerca de un tema, hecho, problema o actividad.

- Llegar a ciertas conclusiones generales y establecer los diversos enfoques que pueden darse a un mismo hecho o tema.

- Incrementar la información de los participantes a través de aportes múltiples.

- Desarrollar el espíritu participativo de los miembros.

Un protagonista muy importante en el foro es el moderador quien debe tener las siguientes características:

- Buena voz y correcta dicción.

- Ha de ser diestro y rápido en su trabajo.

- Prudente y diplomático en ciertas circunstancias. 
- Cordial en todo momento.

- Sereno.

- Seguro de sí mismo.

- Estimulante de la participación y a la vez oportuno controlador de la misma.

Tener ingenio y sentido del humor, porque esto facilitará mucho el mantenimiento del clima adecuado.

\section{Foro Electrónico}

Los foros actualmente son paginas donde cualquiera puede realizar preguntas abiertas con la finalidad de educar como de auto educarnos por medio de personas que tienen la misma duda como la nuestra y pueda resolverla, lo haga con la finalidad de ayudar a difundir la información por este motivo se desea difundir citas científicas a redireccionarlas a comprender de mejor manera su inquietud. (Brito R., s.f.)

Se utilizaron métodos de investigación científica para la búsqueda de información tales como:

\section{Del nivel teórico:}

Análisis-síntesis: Se aplico en todo el desarrollo de búsqueda información y análisis de la misma con la finalidad de buscar antecedentes para contribuir a la solución del problema actual como es la utilización de herramientas viabilizadoras para la distribución de información de carácter científico en el mundo virtual como es el internet.

Inducción-deducción: se utilizó para ir de lo particular a lo general, de los hechos a las generalizaciones, además de ir de lo general a lo particular, de las afirmaciones generales a las conclusiones particulares.

\section{CONCLUSIONES}

Uno de las conclusiones más destacadas en la investigación realizada es la obtención de información que en la actualidad hay muchas formas de poder publicar información de carácter científico desde un blog en internet hasta en las redes sociales comunes por motivo que la tecnología está al alcance de todas las personas.

El internet en la actualidad ayuda mucho a la difusión de información científica solo hay que tener un poco más de responsabilidad e interacción con la tecnología para que todas las personas puedan tener los resultados de nuestras investigaciones realizadas y de esta forma ayudamos a la democratización del conocimiento científico para lograr una sociedad orientada a las investigaciones y aporten al medio que los rodean.

Dentro de la educación Superior cada vez más se necesita la obtención de información científica como causa del crecimiento de las herramientas tecnológicas actuales que facilitan la manipulación de la red más grande de intercomunicación como es el internet por esta razón en el artículo se analizan las diferentes opciones que tenemos a mano para la publicación y distribución de información científica de nuestra autoría con la finalidad de facilitar y ayudar a profesionales y al público en general.

\section{REFERENCIAS BIBLIOGRAFICAS}

Brito R. (s.f.). Obtenido de https://www.edutec.es/revista/index.php/edutec-e/article/view/532/266

94 UNESUM-Ciencias. Publicación cuatrimestral. Vol. 5, Año 2021, No. 5 (Septiembre-Diciembre) 
Ch., M. d. (2005). LAS TECNOLOGÍAS DE LA INFORMACIÓN Y LA COMUNICACIÓN (TIC) HERRAMIENTAS VIABILIZADORAS. ORBIS N $\quad 1 . \quad$ Obtenido de https://www.redalyc.org/pdf/709/70910105.pdf

Daniel Jiménez Bermejo. (s.f.). Obtenido de https://economipedia.com/definiciones/tecnologias-de-la-informacion-ycomunicacion-tic.html

navarra.es. (25 de Febrero de 2018). Obtenido de Acercate a las tics : https://www.navarra.es/NR/rdonlyres/48F9746B-080C-4DEA-BD95A5B6E01797E1/315641/7Usodedispositivosmoviles.pdf

Stelle, L., \& Woodward, H. (2009). Understanding how student and faculty really use e-books. London. Obtenido de http://conferences.aepic.it/index.php/elpub/elpub2009/paper/viewPDFInterstitial/79/36

UNI>ERSIA. (12 de 22 de 2019). Obtenido de UNI>ERSIA: https://www.universia.net/ar/actualidad/orientacionacademica/cuales-son-diversos-tipos-investigacion-cientifica-sus-caracteristicas-1167437.html

Victoria Espinosa Santos. (s.f.). Obtenido de https://scielo.conicyt.cl/scielo.php?script=sci_arttext\&pid=S071834292010000300001

VIU. (Marzo de 21 de 2018). Obtenido de https://www.universidadviu.com/15-herramientas-tic-colaborarcompaneros-profesores/ 
96 UNESUM-Ciencias. Publicación cuatrimestral. Vol. 5, Año 2021, No. 5 (Septiembre-Diciembre) 